\title{
Being on the alert for continuously evolving influenza viruses: Early detection and rapid intervention of newly emerging viruses
}

Author: Mulugeta Berhanu, Ethiopian Agricultural Transformation Agency

Email: mulugetach100@gmail.com; Tel: +251921433836

\begin{abstract}
Influenza viruses cause a major risk to human and animal health. Influenza virus capable of undergoing continuous evolution due to lack of proofreading mechanism during replication and high rate of mutation in their surface glycoproteins. Early detection and rapid intervention are crucial to mitigate the adverse outcomes of newly emerging influenza viruses. Evolution is a serious impediment to antiviral drugs and host immune system. The need for updating flu vaccines arising from perpetual evolution of influenza virus. Vaccines prepared for an earlier form of circulating influenza virus will not provide protection against newly emerging influenza viruses that is why flu vaccines need to be updated regularly. In addition, there is an urgent need for universal influenza vaccines which are capable of protecting against various strains of influenza virus. Hemagglutinin and neuraminidase are the key components of influenza virus which are more prone to mutation. Antigenic variation in influenza virus comes about because of genetic changes in their surface glycoproteins. These changes are known as "antigenic drift" and "antigenic shift". This paper introduces the basics of influenza evolution, highlights the evolutionary history of influenza viruses and discusses how evolution impacts influenza intervention with particular emphasis on the current H5N1 Avian influenza epidemic. This paper also proposes the use of improved adjuvants in flu vaccines to trigger rapid and robust immune responses against newly emerging influenza viruses. Broad-spectrum immunologic adjuvant with ability to induce a wide range of immune responses can be used to develop an effective vaccines against newly emerging flu strains. Generally, this article illustrates how influenza virus undergoes evolution and presents ways of intervening continuously evolving influenza viruses.
\end{abstract}

Keywords: Antigenic drift, Antigenic shift, Avian influenza, Early detection, Evolution, Flu strains, Hemagglutinin, H5N1, Influenza, Neuraminidase, Rapid intervention, Surface glycoproteins, Universal influenza vaccines 


\section{Introduction}

Influenza is rapidly evolving RNA virus which belongs to family Orthomyxoviridae. Influenza virus evolution is driven by antigenic shift and antigenic drift in surface glycoproteins: HA (hemagglutinin) and NA (neuraminidase). Influenza virus constantly changes through mutation and producing new variants. Early detection coupled with rapid intervention plays a critical role in controlling newly emerging influenza viruses before spreading internationally. Currently, various diagnostic tests are available for detection of influenza viruses. However, accurate, easy and fast diagnostic tests are still in demand.

Influenza viruses are divided into three genera, namely influenza type A, B and C. Influenza type " $A$ " is capable of infecting a wide range of hosts whereas type " $\mathrm{B}$ " and " $\mathrm{C}$ " infect a narrow range of hosts. Mutation is most common within the type "A" virus. Influenza virus is characterized by a segmented genome and absence of proofreading mechanism which contributes to continuous evolution of the virus.

Influenza pandemics can have far reaching and long lasting impacts on the public health. Influenza is a devastating viral disease which can be prevented by vaccination. Vaccines play irreplaceable role in protecting the entire global community from continuously evolving influenza viruses which can cause pandemics and epidemics. Vaccines should be updated to induce robust immune response against new flu strains. Updating vaccines by using appropriate antigens which can trigger immune response against new flu strains is crucial to control continuously evolving influenza viruses which can cause pandemics and epidemics. Vaccine with improved adjuvants can be used to overcome newly emerging influenza viruses. It is important to use adjuvants with ability to induce a wide range of immune responses to enhance efficacy of flu vaccines. 


\section{Influenza Virus}

Influenza viruses (family Orthomyxoviridae) are enveloped negative sense single stranded RNA viruses with segmented genomes containing seven to eight gene segments (Palese, and Shaw, 2007; Wright et al., 2007). The influenza viruses comprise three genera in the family Orthomyxoviridae. The genera are commonly referred to as influenza "types "A, B. and C. Influenza viruses are classified into types $\mathrm{A}, \mathrm{B}$ or $\mathrm{C}$ based on antigenic differences of the NP (Wright and Webster, 2001).

Of the 3 types of influenza viruses (A, B. and C) only influenza A viruses are established in animals other than humans (Neumann.and Kawaoka, 2006). Influenza A viruses infect a wide variety of birds, primarily waterfowl, shorebirds, and gulls. Avian influenza A viruses are thought to be the ancestors of influenza A strains currently circulating in swine, horses and humans. The origins of influenza types $\mathrm{B}$ and $\mathrm{C}$, which infect only humans, are unknown (Krossoy el al, 1999).

Influenza is currently the greatest pandemic disease threat to humankind (Gatherer.2009). Influenza B viruses have been isolated from humans, seals and influenza $C$ viruses have been isolated from humans, and swine and usually only causes mild disease in the upper respiratory tract. Influenza B viruses can cause a wide variety of disease, but generally clinical symptoms are similar to those of IAV (Baigent, and McCauley. 2003). Although influenza A viruses are best known for the annual epidemics and occasional pandemics in humans, wild birds form their natural reservoir (Olsen et al., 2006). Whereas only 3 influenza A virus subtypes circulated in humans in the past century, all known subtypes have been isolated from wild birds. Numerous subtypes have also been detected in poultry, generally causing severe disease, mild disease or no disease at all (De Wit and Fouchier, 2008). Several mammals including pigs and horses are also known to be naturally infected by influenza A viruses. Pigs are susceptible to all subtypes of avian influenza viruses in experimental settings (Horimoto and Kawaoka, 2001).

The great majority of influenza viruses are slowly evolving a virulent residents of the intestines of birds. The gene pool of influenza A viruses in aquatic birds provides all the genetic diversity required for the emergence of pandemic influenza viruses for humans, lower animals, and birds. On rare occasion, an avian influenza virus jumps the species barrier and infects a human or other animal. The results can be disastrous: approximately 40 million humans were killed by the 1918 
"Spanish flu." Even the best public health care systems today are not prepared to deal with mortality on this scale (Oxford, 2005).

\subsection{Influenza Virus Structure}

Influenza A virus is roughly spherical (approximately $120 \mathrm{~nm}$ in diameter) with an external envelope. It contains a single negative stranded RNA genome divided into eight segments, encoding 10 proteins. They are haemagglutinin (HA), neuraminidase (NA), nucleoprotein (NP), polymerases (PA, PBJ, PB2), matrix proteins (1\11. M2), and non-structural proteins (NS1 and NS2) (Wright and Webster, 2001). Neuraminidase is a mushroom-shaped tetramer which is the second major surface protein of the virion. It exerts sialolytic enzymatic activity and liberates virus progeny captured at the surface of infected cells during egress. This function prevents viral aggregation during egress and possibly also facilitates the movement of the virus through the mucus layers overlying the targeted epithelial tissues of the host leading to viral attachment (Matrosovich et al.,2004b). This renders the neuraminidase as a target of antiviral agents (Garman, and Laver. 2004). Neuraminidase is not evenly distributed over the virion envelope, as is HA, but are aggregated into patches or caps. Like haemagglutinin, neuraminidase is also highly mutable, with variant selection partly in response to host immune pressure. In addition to $\mathrm{HA}$, and NA, a limited number of M2 proteins are integrated into the virion envelope functioning as a proton channel in relation to virus particle formation and virus uncoating (McCown, and Pekosz, 2006).

Attachment to cell surface proteins is achieved by the virion through mature trimerised viral HA glycoproteins. These recognize distinct terminal sialic acid species ( $\mathrm{N}$-acetyl- or $\mathrm{N}$-glycolyl neuraminic acid), the type of glycosidic linkage to penultimate galactose (alpha 2-3 or alpha 2-6) and the composition of further inner fragments of sialyloligo saccharides present at the host cell surface (Gambaryan et al., 2005). A variety of different sialyloligo saccharides are expressed, which influence tissue, and species specificity in the different hosts of influenza viruses. Adaptation in both the viral HA, and the NA glycoprotein to the specific receptor type(s) of a particular host species is a prerequisite for efficient replication (Banks et al.,2001; Gambaryan el al., 2004; Matrosovich, Krauss, and Webster, 2001). Avian influenza viruses generally show the highest affinities for alpha 2-3 linked sialic acid as this is the dominant receptor type in 
epithelial tissues of endoderrnic origin (gut, lung) in those birds that are targeted by these viruses (Gambaryan et al.,2005; Kim el al., 2005).

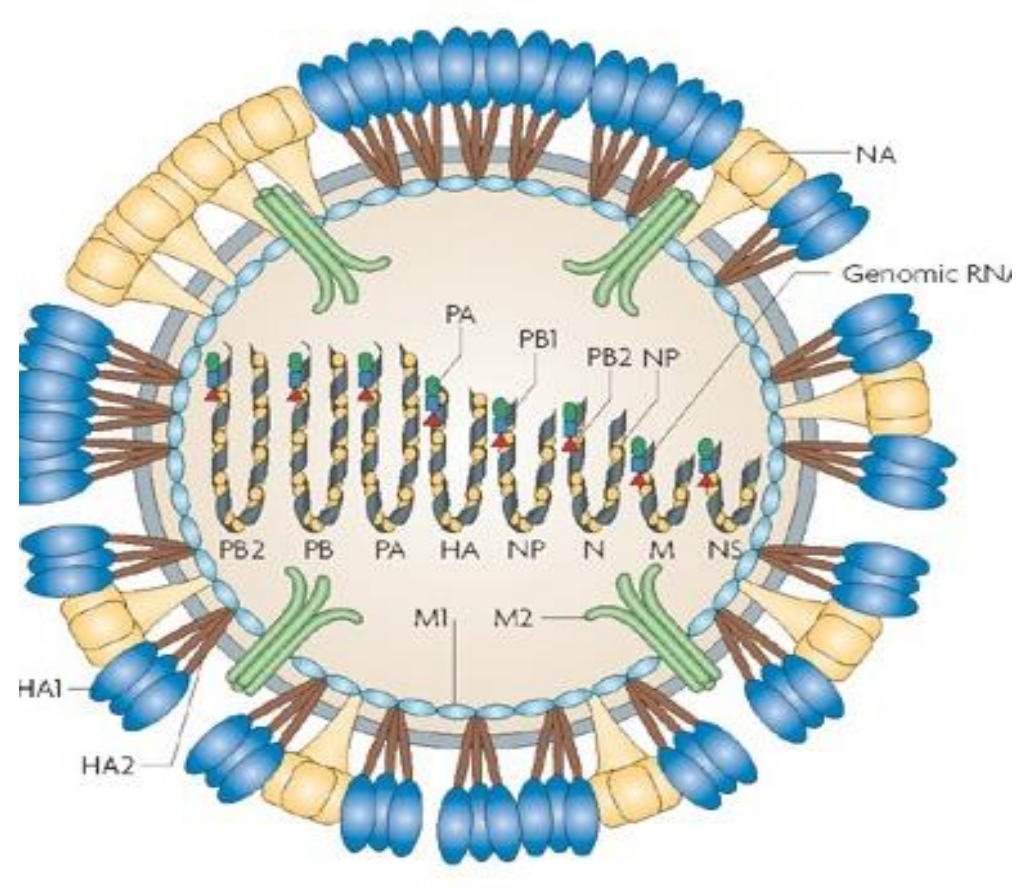

Figure.1. A schematic presentation of influenza A virus. The surface glycoproteins hemagglurinin (HA), and neuraminidase (NA), as well as the ion channel matrix 2 (M2) are located on the surface of the virion. On the inside of the membrane, the matrix (MI) protein forms a matrix holding the viral ribonucleo proteins (vRNPs) containing the viral polymerase complex (PA, PB1 and PB2), and NP, as well as small amounts of NEP.

Source: (Karlsson et al., 2008). 


\subsection{Influenza Virus Genome}

The influenza A virus genome contains eight negative sense RNA segments. The viral mRNAs from segments 1 to 6 are monocistronic while viral mRNAs derived from segments 7or 8 are spliced to form mRNAs coding for two proteins. The sizes of the viral RNA segments and the proteins encoded are summarized in Table 1. Of these proteins, only the NS 1 protein from segment 8 (NS segment) is a non-structural protein (Lamb and Krug, 2001).

Table 1: Influenza A virus genome RNA segments

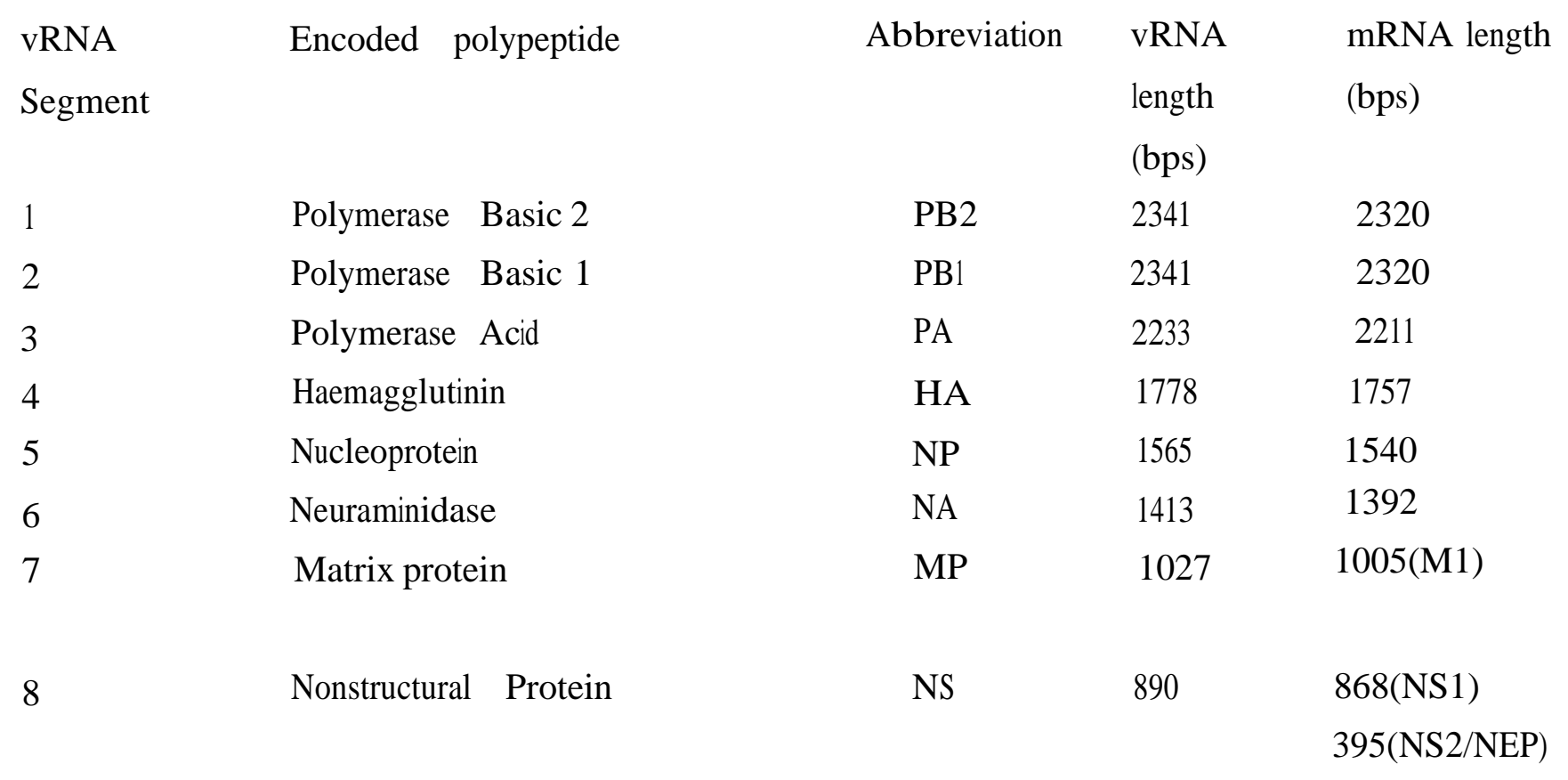

Source: (Lamb and Krug, 2001)

\subsubsection{Segment 1- basic polymerase protein 2 (pb2)}

The first segment of influenza A viruses encodes a $2.3 \mathrm{~Kb}$ protein that forms part of the influenza viral polymerase subunit named PB2. Studies have shown that PB2, PB 1, PA and NP form the minimum set of proteins required for viral transcription and replication (Honda el al.,2002).The PB2 protein specifically plays a role in generating 5'-capped RNA fragments from cellular premRNA molecules that are used as primers for viral transcription (Guilligay et al.,2008). Recently, functional analysis of PB2 protein has shown that this polymerase subunit contains a novel binding site for PB 1 subunit and two regions for binding nucleoprotein (NP) with regulatory interactions potential (Poole et al., 2004). The PB2 gene is reported to play a key role in the 
adaptation of a virus that infects birds to one that infects humans. In particular, it has been reported that the amino acid at position 627 is critical for this adaptation (Van Hoeven et al., 2009). Viruses that infect birds typically have a glutamic acid (E) in this position. On the other hand, influenza A viruses that are fully adapted to humans usually have a lysine $(\mathrm{K})$ at this position. This same position in PB2 appears to be key in determining the lethality of flu viruses (Hatta et al.,2001).

\subsubsection{Segment 2- basic polymerase protein 1 (PB1)}

The PB1 subunit plays a key role in both the assembly of the three polymerase protein subunits, and serves the catalytic function of RNA polymerization. It has been proposed that the catalytic specificity of PB 1 subunit is modulated to the transcriptase by binding PB2 or the replicase by interaction with PA (Honda et al.,2002).

\subsubsection{Segment 3-acidic polymerase protein $(P A)$}

Recent studies however have shown that PA is separable by trypsinization into a large carboxy terminal domain, the crystal structure of which has recently been reported (Obayashi et al.,2008), and a small N-terminal domain, which contains residues important for protein stability, promoter binding, cap-binding, and endonuclease activity of the polymerase complex (Hara et al.,2006).

The viral endonuclease activity which is critical for synthesizing viral mRNA 's have previously been thought to reside in the PB2 or PB1 (Li et al.,2001) subunits. However, recent biochemical, and structural studies have shown that the amino-terminal 209 residues of the PA subunit contain the endonuclease active site and not PB2 (Dias el al.,2009). As such, during transcription, the PB2 subunit binds the 5', 7-methylguanosine cap of a host pre mRNA molecule, which is subsequently cleaved 10--15 nucleotides downstream by the PA endonuclease. The resulting short capped RNA

primers used to initiate polymerization by the RNA-dependent RNA polymerase of the PB1 subunit using 5'- and 3'-bound vRNA as template, resulting in capped, polyadenylated, chimeric mRNA molecules that are exported to the cytoplasm for translation into viral proteins (Boivin et al., 2010$)$.

\subsubsection{Segment 4-haemagglutinin (HA)}

The HA is synthesized as a precursor polypeptide, HA0 (Lamb and Krug, 2001). The generated HA 1 surface subunit mediates the binding to cell surface sialic acid receptors and the HA2 trans 
membrane subunit that mediates membrane fusion between viral and endosomal membranes after endocytosis (Skehel and Wiley, 2000). The specificity of the interaction of HA with sialic acid (SIA), the cellular receptor, largely explains the host ranges of influenza A viruses (Taubenberger and Kash, 2010).

\subsubsection{Segment 5-Nucleoprotein (NP)}

Nucleoprotein, which is an essential component for transcription and replication is encoded by segment 5 of influenza A viruses. Nucleoprotein is distinct among influenza A, B. and C viruses, and is recognized as one of the type-specific viral antigens. It encodes a protein with approximately 500 amino acids; it plays an important role in assembly, and budding of influenza virus, and has a putative role in host range (Ruigrok et al.,2010). To achieve its biological functions, recent studies have revealed that the NP is capable of interacting with various host proteins (Taubenberger and Kash, 2010; Wang et al., 2009).

\section{2. 6. Segment 6 - Neuraminidase (NA)}

Strong binding to receptor analogs on mucins, cilia, and cellular epithelia would inhibit virus access to functional receptors on surface membrane of target cells. Therefore, neuraminidase is important for releasing viruses from the decoy receptor, and plays an essential role of virus entry in the early stage of infection (Matrosovich et al., 2004a). It is for this reason that NA is a favorable target for antiviral drugs aiming at reducing its sialidase enzymatic activity and thus release of progeny virions (Suzuki et al., 2005).

\subsubsection{Segment 7-Matrix proteins (Ml and M2)}

It is found to interact with the viral surface glycoproteins, anti the RNP complex (Taubenberger, and Kash, 2010).

\subsubsection{Segment 8-Non-structural proteins (NS1 and NS2)}

Non-structural protein 1 is the only non-structural protein of influenza virus, and is found mainly in the host cell nucleus. It is a multifunctional protein involved in nuclear exportation of mRNA, post-transcriptional regulation, and inhibition of cellular interferon response (Hale et al.,2008). Recently, NS1 protein was found to be responsible for the unusual severity of H5N1 diseases by inducing exaggerated pro-inflammatory cytokine responses (Cheung et al., 2002). NS2 protein 
exists in low amounts, and binds to M1 protein in virion. The protein may promote the formation of a stable export complex of new viral RNP (Neumann et al., 2000). In association with the matrix protein 1 (M1), it interacts with cellular export factor (CEF1), and mediates the nuclear export of viral riboneuclo protein (vRNP) complexes by connecting the cellular export machinery with vRNPs (Neumann el al.,2000).

\subsection{Diversity of influenza A virus subtypes}

Influenza A viruses are subtyped according to their surface glycoproteins HA, and NA, and to date, 18HA, and 11NA subtypes have been isolated, where the H17N10, and H18N11 are the most recently discovered subtypes and have only been isolated from bats (Fouchier et al.,2005; Tong et al.,2013; Tong et al., 2012). HA subtypes are further divided into two groups based on phylogenetic relationship, where group 1 includes $\mathrm{H1}, \mathrm{H} 2$, H5, H6, H8, H9, H11, H12, H13, H16, H17 and H18; group 2 includes H3, H4, H7, H10, H14 and H15 (Wu el al.. 2014). Also NA can be divided into two groups, here group 1 contains N1, N4, N5 and N8 and group 2 contains N2, N3, N6, N7 and N9 (Russell et al..2006), recently N10 and N 11 have been proposed to form group 3 (Wu et al., 2014). Several mammals including pigs and horses are known to be naturally infected by influenza A viruses. Pigs are susceptible to all subtypes of avian influenza viruses in experimental settings (Horimoto and Kawaoka, 2001). A new subtype of influenza A virus is introduced into the human population from swine or birds that is able to spread efficiently from human to human, causing a pandemic. This process is called antigenic shift, and may be the result of adaptation of a fully avian virus to humans (Tumpey et al., 2005).

\section{Influenza Evolution}

Influenza is the paradigm of a viral disease in which continued evolution of the virus is of paramount importance for annual epidemics and occasional pandemics of disease in humans (Robert, 1992). Influenza evolution refers to antigenic variation of the influenza virus which can be divided into two phenomenons. namely antigenic shift and antigenic drift. Both antigenic drift and antigenic shift allow for the virus to evade the host's immune response, and rapidly adapt to new hosts (Caron et al., 2009; Suzuki, 2006). Antigenic shift can occur when a host is co-infected with two or more different IAVs, and gene segments are interchanged during virion packing. This event is termed reassortment and is defined by the exchange of intact segments between two differing influenza A viruses (Brown, 2000). The antigenicity of influenza viruses changes both 
gradually by point mutation (antigenic drift), and drastically by genetic reassortrnent (antigenic shift) (Wright and Webster, 2001).

The influenza A virus is divided into subtypes based on differences in the surface proteins hemagglutinin (HA) and neuraminidase (NA), which are targets of the human immune system. Antigenic variants or immunologically distinct strains of A/H1N1, A/H3N2 and Type B have continued to emerge since its introduction into humans (Schweiger et al., 2002).

Influenza A viruses have been able to adapt stably to a wide variety of animals, including avian and mammalian species and novel human-adapted IAVs have emerged to cause pandemics several times in the last 100 years. Each of the viruses causing these pandemics has emerged in a different way, making generalizations about zoonotic IAV adaptation to humans difficult. As data have accumulated, it has become clear that IAV host switch events are polygenic, and represent different solutions to the common problem of replication and transmission in a host (Taubenberger, and Morens, 2009).

\section{Evolutionary history and phylogenetics}

During the past century, three pandemics occurred. The first of these is the so called 'Spanish influenza' which occurred in 1918, and which is considered as the greatest natural disaster of the 20th century. Approximately 40 million humans were killed by the 1918 "Spanish flu." This pandemic occurred as a result of the introduction of an influenza A virus of the H1N1 subtype probably derived from an unidentified avian-like precursor virus which became adapted to mammals (Morens et al.,2009).

The second such pandemic occurred in 1957 due to the introduction of the H2N2 subtype causing the so called 'Asian influenza'. This pandemic virus emerged by reassortment between the viruses in humans at that time and the virus from some aquatic avian reservoir killing an estimated 2 million people worldwide (Morens et al., 2009). 
The last pandemic of the 20th century occurred in 1968, and was due to influenza A H3N2 virus subtype derived from both human and avian viruses. This pandemic was relatively mild (De Wit, and Fouchier, 2008). The PB 1 gene was itself seeded in swine from human (Garten et al.. 2009), and two segments (NA and M) from the Eurasian lineage (Dawood et al., 2009). The amino acid sequence divergence of the 2009 pandemic $\mathrm{H} 1$ from human seasonal influenza H1 is around 20$24 \%$, and for this reason, it is being considered as halfway towards a new serotype (Gatherer, 2009). Results of virological surveillance conducted in Hong Kong live poultry markets before the depopulation in 1997 showed that several subtypes of avian influenza viruses were co-circulating with the H5N1viruses during that time. Avian influenza has received world-wide attention as the highly pathogenic H5N1 virus progressively spread to poultry, and wild bird populations across Asia, Africa, the Middle East and Europe, and unexpectedly 'traversed inter-class barriers' (FAO, 2006; Perkins and Swayne, 2003) when transmitted from birds to mammals such as humans, pigs, and cats (FAO, 2006; Kuiken et al.,2004; WHO, 2005). Although not an entirely unexpected event (Hayden and Croisier, 2005; Koopmans et al., 2004), the substantial number of documented human cases associated with severe disease and fatalities raised serious concerns about the pandemic potential of the H5N1 strain (Webster et al., 2006).

Looking at the past phylogenetic relationships of the influenza virus can help lead to information regarding treatment, resistance, vaccine strain selection, and of future possible influenza strains. By looking at how previous strains have evolved, and gained new traits, the information can be applied to predict how current strains can evolve, and even how novel strains might come about (Luksza et al.,2014). Another use of phylogeny for predicting future viral dangers would be through using various lineages may continue their presence, and reassert indicating the importance of a complete-genome approach to determine new influenza strains and future epidemics (Holmes et al.,2005, Vana, 2008). 
By studying how past strains have evolved while spreading to different geographic regions can allow scientists to predict how a strain might accumulate new mutations through its geographic distribution, and the information could be used to protect different populations (Viboud, 2003).

Phylogenetic analysis of two of the most commonly isolated viruses (H9N2 and H6N1) showed that these two viruses are closely related to the 1997 H5N1 virus (Guan et al., 2000; Hoffmann et al.,2000).Estimation of phylogenetic trees has the advantage, other than predicting evolutionary relationship between sequences, that they can also be used for the prediction of selection on the genes (Yang, and Nielsen, 2000), as well as for the estimation of evolutionary rates, and time of divergence in relation to the most recent common ancestor for a group of sequences (Holder and Lewis, 2003).

Phylogenetic trees constructed using influenza sequence data are a natural way to monitor the genetic changes associated with antigenic drift. The World Health Organization (WHO) and Global Influenza Surveillance Network have conducted influenza surveillance worldwide since 1952. Antigenic characterization initially formed the basis of surveillance: gene sequencing was added as a surveillance tool in the mid-1980s. These efforts have produced a wealth of data for evolutionary analysis.

Figure 2 shows phylogenies constructed using the HA1 domain of the HA gene of human influenza A subtypes H3N2, and H1N1 and of influenza B (Bush et al., 2004). The slender "trunk" of the $\mathrm{H} 3$ tree in an especially characteristic reflection of the serial replacement of strains that result from antigenic drift. Although brief co-circulation of closely related H3 lineages is not uncommon, the H3 tree appears linear because the average survival time of side lineages is only 1.6 years (Fitch et al., 1977).

The $\mathrm{H} 1$ tree is similar to that of $\mathrm{H} 3$, although recent bifurcation of the main trunk has resulted in several extant lineages. It remains to be seen whether more than one will persist. The influenza B virus was first isolated from humans in 1940; its evolutionary history prior to that point in time is unknown. Although limited sequence data exist prior to the mid-1980s, phylogenetic analysis shows a split into two distinct B lineages sometime in the early 1970s. One lineage circulated globally throughout the 1990s; the other lineage was restricted to Asia. In the last few years, the restricted lineage has reappeared. Re-emergence may have been due to the accumulation of a large 
cohort of immunologically naive children. However, genetic change, including between lineage reassortment, occurred during this time as well (Lindstrom et al., 2004).

If both influenza B lineages persist, it may be necessary to add an additional influenza B virus to the current vaccine formulation. The processes responsible for these phylogenetic patterns are not well understood (Ferguson et al., 2003). Studied the interaction between ecological, and immunological factors affecting drift using a mathematical model that incorporated both realistic epidemiological dynamics, and viral evolution at the sequence level. Matching of model output to phylogenetic patterns suggested that short-lived strain-transcending immunity, such that infection by one strain of influenza prevented reinjection by any strain during the next month or so, was essential to restrict viral diversity, and linearize the tree. This result is intriguing, but the precise immunological factors responsible remain to be identified. It is easy to draw intuitive but incorrect inferences from quick visual inspection of an influenza tree. For instance, one might think, given the linear phylogeny of the H3N2 tree that each subsequent influenza epidemic evolves from the previous epidemic strain. This is not always the case. For example, the A/Singapore/1/86-like H1Nl viruses that caused the 1986-1987 epidemic did not evolve from the previous epidemic strain, the A/Chile/1/83-like viruses. They belonged to a separate lineage; the ancestor of both strains was a virus that had circulated much earlier in China.

It is also a mistake to assume that the sizes of the clades (genetic clusters) on the trees in Figure 2 reflect the frequencies of these genotypes in nature, or the severity of the disease that they caused. Surveillance by the WHO, currently the main source of influenza sequence data, is purpose fully biased toward sequencing viruses that differ antigenically from the current epidemic strain on the basis of the hemagglutination inhibition test. As a result of this intentional sampling bias, HA genotypes at high frequencies in nature may in fact not be most frequent in Gen Bank, particularly in years with few samples. Assuming otherwise will inevitably lead to erroneous results under current WHO sampling protocols (Plotkin et al.,2002). 


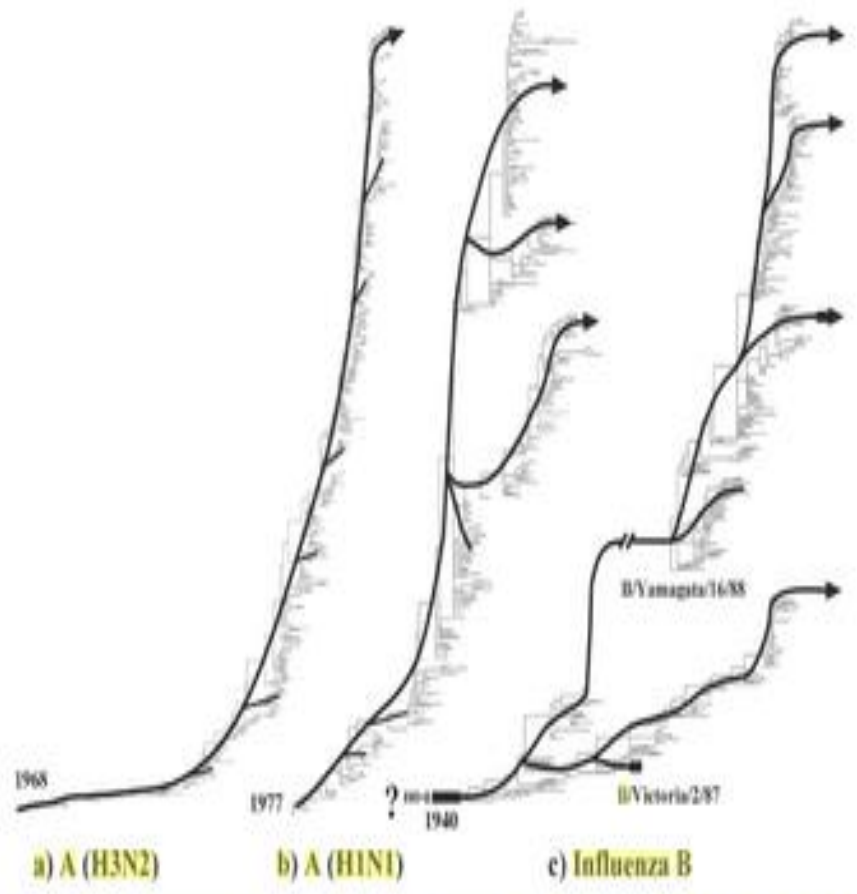

Figure 2. Influenza phylogenies. Phylogenetic trees showing the evolution of the HA1 of influenza Subtypes H3N2 (a), and H1N1 (b), and influenza B. Dark lines are superimposed to indicate some of the major genetic lineages. As explained in the text, because of non-random sampling the relative size of various branches is not an accurate reflection of the frequency of those genetic lineages in nature. Lines ending in arrows indicate extant lineages.

\subsection{Mechanisms and causes of evolution}

The evolution of influenza A virus is driven by the high rate of mutations, and the ability to reassort gene segments (Boni,2008). Influenza A viruses are evolutionarily dynamic viruses, and have high mutation rates (ranging from approximately $1 \times 10-3$ to $8 \times 10-3$ substitutions per site per year). Random mutations can be rapidly selected for or against depending upon the evolutionary pressures applied. including novel host environment (Landolt, and Olsen, 2007), response to preexisting immunity leading to antigenic drift (Smith et al.. 2004), or antiviral drug pressure leading to resistance (Ong and Hayden, 2007). Because of its high rate of mutation combined with the lack of error correcting mechanisms during replication influenza virus can 
easily generate different phenotypes that have the ability to survive within its host and infect others.

Continuing evolution is most prominent in the surface glycoproteins of influenza viruses but also occurs in each of the eight gene segments of both type A and type B influenza viruses. The variability results from accumulation of molecular changes in the eight RNA segments that can occur by a number of different mechanisms including (i) point mutations (antigenic drift), (ii) gene reassortment (genetic shift), (iii) defective-interfering particles, and (iv) RNA recombination. Each of these mechanisms may contribute to the evolution of influenza viruses (Steinhauser. 1987). Sequencing of the whole influenza virus genome facilitates comparison and understanding of the evolutionary dynamics of circulating viruses and the prediction of potential evolution events that are likely to result in new strains (Greninger et al., 2010).

Two common mechanisms by which viruses evolve are Antigenic drift, and Antigenic shift (Chen, and Holmes, 2006). Both antigenic drift and antigenic shift allow for the virus to evade the host's immune response and rapidly adapt to new hosts (Caron et al., 2009: Suzuki, 2006).

\subsubsection{Antigenic shift}

Antigenic shift occurs when complete gene segments are exchanged among different subtypes of influenza viruses within a host cell, resulting in what effectively amounts to a whole new influenza virus genome (Caron et al.. 2009: Suzuki, 2006).

Antigenic shift is sudden and profound change in antigenic determinants, i.e. a switch of Hand/or $\mathrm{N}$ subtypes, within a single replication cycle. This occurs in a cell which is simultaneously infected by two or more influenza A viruses of different subtypes. Since the distribution of replicated viral genomic segments into budding virus progeny occurs independently from the subtype origin of each segment, replication-competent progeny carrying genetic information of different parental viruses (so-called re-assortants) may spring up and show substantial differences from progenitor viruses in their epidemiological behavior (Webster and Hulse. 2004). 
Re-circulation of existing subtypes is another form of antigenic shift in the susceptible population. Evidence for this theory comes from sero-epidemiological studies of antibody to influenza viruses in sera taken at different times from subjects of different ages in population. Antibody to a human influenza A subtype was often found in the sera of elderly persons taken years before the appearance of the same subtype as a cause of pandemic infection. It was suggested that all influenza A subtypes exist in nature, and emerge when the antibody status of the population has fallen to levels which allow new infection (Kilbourne et al., 1990). In this kind of antigenic shift, influenza viruses exploit different strategies to escape immune surveillance, including the introduction of mutations in cytotoxic T-lymphocyte (CTL) epitopes (Voeten et al.,2000). Antigenic shift occurs when complete gene segments are exchanged among different subtypes of influenza viruses within a host cell, resulting in what effectively amounts to a whole new influenza virus genome (Caron et al., 2009; Suzuki, 2006).

Because the IAV genome consists of eight discrete RNA segments, co-infection of one host cell with two different IAVs can result in progeny viruses containing gene segments of both parental viruses. When this process of genetic reassortment involves the gene segments encoding the HA and/or NA genes it has been termed antigenic shift. There are theoretically 256 (28) possible combinations of the eight gene segments from reassortment between two parental viruses. Reassortment has been shown to be both common, and important in IAV evolution (Dugan et al., 2008: Holmes et al.,2005), and host switch events (Garten et al., 2009).

Homologous recombination is not common in negative sense RNA viruses like IAV (Boni et al .2008). But recombination by template switching has been described, and has played a role in changing the virulence or fitness of some IAVs (Wright et al., 2007). The other mechanism for antigenic shift is the gradual adaptation of animal influenza viruses (avian especially) to human transmission. There is now evidence that this might have happened in the 1918 pandemic that the pandemic virus was directly descended from an avian ancestor (Taubenberger et al.,2005). 


\subsubsection{Antigenic drift}

A slower, more continuous evolutionary process called "antigenic drift" occurs in influenza lineages once they become established in a new host. Antigenic drift is due to the high mutational rate of the polymerase, where the lack of proofreading of the RNA polymerases contribute to a mutation rate of the order of 10-5 to 10-6 substitutions/site/replication and the selective pressure of the immune system of the host. Most nucleotide substitutions are silent, meaning that they do not affect the virus (Nobusawa and Sato, 2006). Antigenic drift consists of the accumulated and continual mutations on surface proteins, resulting in the generation of antigenic variants (Caron et al.. 2009: Suzuki, 2006).

The majority of amino acids residues in the IAV are negatively selected, a process termed purifying selection. This means that substitutions are only accepted under certain selection pressures, since they otherwise would affect viral fitness. When amino acid residues are positively selected, it is termed diversifying selection, and only very few residues are positively selected. The combination of the lack of proofreading of the polymerase, and the immune pressure exerted by the will favor the situation where the virus obtains a substitution that permits its escape from the immune system, and thereby provides a fitness advantage to the virus. The accumulation of these substitutions will eventually lead to the development of an antigenically different virus strain which is more fit, and able to replace existing strains (Zell et al., 2012).

\section{Evolution in various hosts}

\subsection{Influenza evolution in avians}

Two evolutionary models can explain the global pattern of IAV diversity in wild birds, analogous to the allopatric and sympatric models of speciation and it is likely that both have played roles in IAV evolution in wild birds (Dugan et al., 2008). Phylogenetic analyses demonstrate that while all IAV HA subtypes had a common ancestor, the HA subtypes did not originate in a single radiation, and include higher-order clustering. Inter-subtype genetic diversity is high, but intra-subtype diversity is quite low. Hence, the genetic structure of avian IAV HA is characterized by highly divergent subtypes that harbor relatively little internal genetic diversity. This is also the case for the evolution of the 9 NA subtypes. Interestingly, analyses suggest that this diversity reflects a 
very recent origin, with ranges for the most recent common ancestors (TMRCAs) of the different HA subtypes in the period of the last several hundred years (Chen and Holmes, 2010).

The NS gene segment in bird IAV is characterized by a deep divergence between the A and B alleles, strongly suggesting that the two alleles are subject to some form of balancing selection (Dugan et al.,2008). Far less genetic diversity is observed in the 5 remaining IAV gene segments in wild birds (PB2, PB 1, PA, NP and M). Phylogenetic analyses also reveal a clear separation of avian IAV sequences from Eastern, and Western Hemispheres, supporting allopatric evolutionary pressures (Dugan el al., 2008; Munster and Fouchier, 2009).

Mixed infection, and reassortment has also been shown to be extremely common in IAV in wild birds (Wang et al., 2008) with little evidence of genetic linkage among specific segments. The large number of different HA-NA subtype combinations recovered also highlights the frequency of reassortment in avian IAV, and provides little evidence for the elevated fitness of specific HANA combinations. In contrast to the extensive genetic diversity seen in HA, NA and NS, the 5 remaining internal gene segments encode proteins that are highly conserved at the amino acid level, indicating that they are subject to widespread purifying selection. The fitness landscape for these genes is therefore not determined by cross-immunity, but by functional viability with less selective pressure to fix advantageous mutations. Given such strong conservation of amino acid sequence, large-scale reassortment likely involves the exchange of functionally equivalent segments, with little impact on overall fitness. Dugan, et al. hypothesized that IAV in wild birds exists as a large pool of functionally equivalent, and so often inter-changeable, gene segment that form transient genome constellations, without the strong selective pressure to be maintained as linked genomes (Dugan et al., 2008). IAV maintained in wild birds have been associated with stable host switch events to novel hosts including domestic gallinaceous poultry, horses, swine and humans leading to the emergence of viral lineages transmissible in the new host. Adaptation to domestic poultry species is the most frequent (Wright et al., 2007).

Stable host switching likely involves the acquisition of a number of mutations depending on the virus, and the species that serve to separate an individual, clonally-derived IAVs strain from the large wild bird IAV gene pool. Because adaptation to a new host likely limits the ability of these viruses to return to the wild bird IAV gene pool (Swayne, 2007), these emergent viruses must evolve as distinct eight-segment genome constellations within the new host (Dugan et al., 2008; 
Taubenberger and Morens, 2009). Sporadically, strains of poultry-adapted H5 or H7 IAV evolve into HPAI usually through acquisition of an insertional mutation resulting in a polybasic amino acid cleavage site within the HA (Wright et al., 2007).

The current panzootic of Asian-lineage HPAI H5N 1 appears to be unique in the era of modern influenza virology (Webster et al., 2007). The molecular features of host adaptation to domestic gallinaceous poultry are not yet fully elucidated, but include positive selection for mutations in both HA and NA (Campitelli et al., 2004; Perez et al., 2003), and viral RNP proteins (Wasilenko et al., 2008). Influenza A viruses isolated from domestic poultry generally maintain an HA receptor binding specificity for alpha 2, 3-SA (Wright et al.. 2007). Neuraminidase stalk deletion has been associated with reduced enzymatic activity of the NA (Baigent and McCauley, 2001). and may be a compensatory adaptive change to reduced HA receptor binding activity of wild bird IAV adapted to replicate in the respiratory tract of poultry.

\subsection{Influenza evolution in equine and swine}

Two IAV H7N7 and H3N8 have been emerged in equines. Equine H7N7 is now likely extinct, but H3N8 equine IAV lineage, first detected in the early 1960's, continues to circulate enzootically, and cause a high disease and economic burden on the horse industry. In 1989 an independent, avian-like H3N8 virus was isolated following an epizootic in horses in China, but this lineage has not persisted. Available phylogenetic evidence suggests that all these equine lineages were derived from avian IAV (Horimoto and Kawaoka, 2001 ). Unlike swine IAV, equine IAV has been recognized for hundreds of years (Taubenberger and Morens, 2009), interestingly often in close association with human IAV epidemics or pandemics. In the virologic era, the first IAV isolated from horses occurred in an epizootic in 1956.

In March and early April 2009; new swine-origin influenza A (H1N1) virus (S-OIV) emerged in Mexico and the United States (CDC, 2009). Since then, the virus (later renamed influenza A (HIN1) pdm09 virus) spread rapidly across the world by human-to-human transmission. On the 1 lth of June 2009, the World Health Organization declared a global pandemic of 2009 H1N1 infection making it the first pandemic of the 21st century (WHO, 2009). By April 2010, there were 18,500 laboratory confirmed deaths with recent studies estimating a higher figure of 201,200 deaths (Viboud and Simonsen, 2012). 
The outbreak strain was identified as a swine origin influenza virus that resulted from a reassortment of two previously circulating strains. The new strain contains six segments (PB2, PBl, PA, HA, NP, and NS) that were similar to the ones previously found in triple-reassortant swine influenza viruses circulating in pigs in North America (Dawood et al., 2009). From 1998, several different lineages of 'triple' reassortant viruses of H3N2, H1N2 and H1N1 subtypes, containing genes from the classical swine $\mathrm{H} 1 \mathrm{~N} 1$, human $\mathrm{H} 3 \mathrm{~N} 2$ and avian IAVs, emerged to cause enzootic disease in pigs in the U. S. and globally (Olsen, 2002).

In Europe, a novel lineage of HINl emerged in the late 1970's by adaptation of an avian IAV to swine leading to enzootic disease in Eurasia (Dunham et al., 2009). Other fully avian or fully human IAV-derived isolates or reassortant viruses containing genes of avian, swine and/or human IAV origin, have been associated with less widespread disease in swine (Reperant et al.,2009).

\subsection{Influenza evolution in humans}

The most salient feature of influenza evolution in humans is its antigenic drift. This process is characterized by structural changes in the virus's B-cell epitomes' and ultimately results in the ability of the virus to evade immune recognition and thereby re-infect previously infected hosts (Yuan and Koelle, 2013). The emergence of the pandemic H1N1 (H1Nlpdm09) virus in 2009 in humans followed by reverse zoonotic events, introduced yet another H1N1 virus in pigs in many countries (Pereda et al., 2010; Vijaykrishna et al., 2010). Only three HA subtypes of IAV (H1N1, $\mathrm{H} 2 \mathrm{~N} 2$ and H3N2) have caused epidemics in humans in modern times. The most severe pandemic in the past century was caused by the H1N1 subtype in 1918, also known as "the Spanish flu", which resulted in approximately 546,000 deaths alone in the United States (Taubenberger and Morens, 2006).

In 1957, a pandemic was caused by an IAV of H2N2 subtype, known as the "Asian flu" due to its origin in China. The H2N2 subtype was replaced by the H3N2 subtype in 1968, which was the cause of the next pandemic known as the "Hong Kong flu". In 1977, a re-emergence of a descendant of the $1918 \mathrm{H} 1 \mathrm{Nl}$ IAV occurred, which was found to be genetically similar to the viruses that circulated before 1957. Since it was thought highly unlikely that a virus could have circulated for 20 years without being detected or having accumulated mutations, it was therefore suggested that the re-emergence had to be caused by an accidental release of a frozen version of an early 1950 strain (Nakajima et al., 1981; Taubenberger and Kash, 2010). 
In 2009 a new pandemic HIN1 virus (H1N1pdm09) occurred which differed from the earlier known H1N1 viruses (Smith et al., 2009) and since then, this IAV has gradually replaced the seasonal H1N1 virus and began co-circulating with H3N2 causing seasonal influenza epidemics in humans (Neumann and Kawaoka, 2011).

\section{Methods for detecting influenza virus}

Public health is being attacked with influenza containing new genes from a genetic assortment between animals and humankind. Unfortunately, the population does not have immunity for mutant genes and is attacked in every viral outbreak season. For these reasons, fast and accurate devices are in high demand. As currently used methods like Rapid Influenza Diagnostic Tests lack specificity, time and cost-savings, new methods are being developed (Dziąbowska K et al., 2018).

Methods for detection of influenza viruses are conventional serological methods as well as the advanced methods of molecular biology and biosensors. All these methods are based on different parameters and have different targets but the goal is to improve specificity and increase sensitivity. Amongst the molecular methods, real-time polymerase chain reaction (RT-PCR) is considered a gold standard test due to its many advantages whereas a number of other molecular methods are time-consuming, complex to perform or lack specificity (Ravina et al., 2021).

\section{Evolution and Intervention}

Vaccine has played a critical role in protecting the global community from various infectious diseases (Berhanu, M. 2021). Vaccines are currently available for the prevention of human influenza and also for some subtypes of avian influenza. Two classes of antiviral drugs, although not widely used, have been approved for influenza prophylaxis and treatment. The human influenza vaccine first developed in 1947 is currently a trivalent formulation containing one influenza B virus and one virus each from influenza A subtypes H3N2 and H1N1. 
To keep track of the evolution of the virus, annual update to the influenza vaccine composition is needed in order to provide a vaccine induced immunity to the general public (Boni, 2008). Antigenic drift requires that vaccine strains be updated almost yearly, selection of viral strains for inclusion in the vaccine is based on antigenic, genetic, and epidemiological data and practical issues such as the availability of high growth reassortments suitable for vaccine production. Reverse genetic and cell culture techniques are now being applied to the production of an H5N1 vaccine. If successful these methods may eventually be adapted for the production of the yearly vaccine as well. This would provide greater flexibility and standardization in vaccine production and also allow for a more rapid vaccine response to antigenic drift. The need for frequent vaccine updates might also be reduced by the development of a vaccine eliciting protective antibodies against a more evolutionary stable part of the virus than the HA (De Fillette et al., 2005).

One of the most studied and developed of these universal vaccines are those that target the extracellular portion of the M2 protein (M2e) (De jong et al., 2000). M2e is not the only conserved epitope between influenza viruses, and a number of other proteins have been targeted. These include nucleoprotein, and polymerase proteins through T-cell mediated approaches and also more conserved domains of HA (Dowdle et al.,1997). In 2011 there was some research success towards a universal vaccine that produces antibodies against proteins on the viral coat which mutate less rapidly, and thus a single shot could potentially provide longer-lasting protection (Dowdle et al., 1999).

Two families of antiviral drugs are currently used to treat human influenza virus infections. Oseltamivir (Tamiflu; Roche) and zanamivir (Relenza: GlaxoSmithKline) inhibit the neuraminidase activity of the NA protein, thus blocking release of the newly formed virions from infected cells. The adamantanes (amantadine, and rimantadine) inhibit the ability of the viral ion channel protein $\mathrm{M} 2$ to exchange $\mathrm{H}+$ in order to lower the $\mathrm{pH}$ inside the virus, a step that is needed for viral uncoating during entry into the host cell. Although these antiviral therapies are efficacious against the current influenza virus strains, their use can result in the selection of resistant viruses, and resistant strains do occur in nature. This emphasizes the great need for additional broad-spectrum therapeutic approaches (Zuckerman, 2004). 


\section{Conclusions and Recommendations}

This paper describes how influenza virus undergoes evolution and presents ways of intervening continuously evolving influenza viruses. In this article, the past phylogenetic relationships of the influenza virus, evolution in various hosts, mechanism and causes of evolution were discussed. Comprehensive understanding of evolutionary history of influenza viruses can help us to predict the future possible influenza strains. Influenza virus undergoes perpetual evolution for a couple of reasons. These are lack of proofreading mechanism during replication and high rate of mutation in their surface glycoproteins. A highly pathogenic flu strain can evolve from a low pathogenic strains. The need for updating flu vaccines arising from perpetual evolution of influenza virus. Early detection and rapid intervention of newly emerging influenza viruses are crucial to prevent future pandemics.

Based on the above conclusive remarks the following recommendations were forwarded:

Further studies should be carried out to halt evolution of a highly pathogenic strain from a low pathogenic strain.

Early detection and rapid response (EDRR) policy should be put into effect to mitigate the adverse outcomes of newly emerging influenza viruses.

Flu vaccines should be updated yearly to control continuously evolving influenza viruses.

Research should be targeted on universal influenza vaccines which are capable of protecting against different strains of influenza virus.

Prophylactic antiviral therapy should be recommended as soon as the pandemic emerges so as to slow down the rate of new infection.

There should be strong collaboration among human health personnel, veterinarians and other relevant stake holders (one health approach) so as to control future influenza epidemics and pandemics. 


\section{References}

Baigent SJ, McCauley JW. (2001): Glycosylation of haemagglutinin and stalk-length of neuraminidase combine to regulate the growth of avian influenza viruses in tissue culture. Virus. Res. 79,177--185.

Banks, J., Speidel, E. S .,Moore, E., Plowright, L., Piccirillo, A., Capua, I., Cordioli, P., Fioretti, A., and Alexander, D. J. (2001): Changes in the haemagglutinin and the neuraminidase genes prior to the emergence of highly pathogenic H7N I avian influenza viruses in Italy. Archives of Virology.146(5), Pp: 963-973.

Berhanu, M. (2021, July 30). Vaccine containing immunologic adjuvants with a wide range of activity to provide protection against COVID-19. https://doi.org/10.31730/osf.io/es9cf

Boni, Maciej F. (2008): Vaccination and antigenic drift in influenza.Vaccine, 26 Supp 13, C 814.

Bush RM, Cox NJ. (2004): Influenza evolution. In Infectious Disease and Host-Pathogen Evolution(ed. K.R. Dronarnraju). CambridgeUniversity Press. Cambridge, UK Pp. 17597.

Campitelli, L., Mogavero. E., De Marco, M.A., Delogu, M., Puzelli, S., Frezza. F .. Facchini. M., Chiapponi, C., Foni, E. andCordioli, P. (2004): Interspecies transmission of an H7N3 influenza virus from wild birds to intensively reared domestic poultry in Italy, Virology. 323,2436

Caron Alexandre Caron, Nicolas Gaidet, Michel de Garine-Wichatitsky, Serge Morand, and Elissa Z. Cameron, (2004): Evolutionary biology, community ecology and avian influenza research. Infect Genet Evol, 9(2): 298-303.

Center for disease control and prevention, (2009): Swine influenza A (H1N1) infection in two children in Southern California, J1.MJVR. Morb. Mortal. Wkly. Rep. 58, 400- 402.

Chen R Holmes EC (2010): Hitchhiking and the population genetic structure of avian influenza virus. J. mol.evol;70:98-105.Chen R, Holmes EC. Avian influenza virus exhibits rapid evolutionary dynamics. Mol. Boil. evol. 2006;23:2336-2341. 
Cheung, C.Y., Poon. L.L., Lau, A.S., Luk. W., Lau. Y.L., Shortridge. K.F., Gordon, S., Guan, Y., Peiris, J.S., (2002): Induction of proinflammatory cytokines in human macrophages by influenza A (HSN J) viruses: a mechanism for the unusual severity of human disease.lancet.360,1831- 1837.

Dawood, F.S., Jain, S., Finelli, L., Shaw, M.W., Lindstrom, S., Garten, R.J., Gubareva, L.V., Xu, X., Bridges, C.B., Uyeki, T.M., (2009): Emergence of a novel swine-origin influenza A (HIN1) virus in humans. N. Engl. J Med. 360, 2605-2615.

De Filette,M., Min Jou, W. and Birkett, A. (2005: Universal influenza A vaccine: optimization of M2 based constructs. Virology. 337,149-61.

De Wit. E. and Fouchier, R.A., (2008): Emerging influenza. J Clin. Viral. 41, 1-6

Dias, A., Bouvier, D., Crepin, T., McCarthy, A.A ., Hart D.J., Baudin, F., Cusack. S., Ruigrok, R.W., (2009): The cap-snatching endonuclease of influenza virus polymerase resides in the PA subunit Nat. 458, 914-91.

Dugan, V.G., Chen, R., Spiro, DJ., Sengamalay, N., Zaborsky, J., Ghedin, E., Nolting, J., Swayne, D.E., Runstadler, J.A. andHapp, G.M. (2008): The evolutionary genetics and emergence of avian influenza viruses in wild birds. PLoSPathog, 4, 176.

Dunham EJ, Dugan VG, Kaser EK, Perkins SE. Brown IH. Holmes EC. Taubenberger JK (2009): Different evolutionary trajectories of European avian-like and classical swine H1N1 influenza A viruses. J Virol.83, 548.

Dziąbowska K, Czaczyk E, Nidzworski D. Detection Methods of Human and Animal Influenza Virus-Current Trends. Biosensors (Basel). 2018;8(4):94. Published 2018 Oct 18. doi:10.3390/bios8040094

Ferguson NM. Galvani AP, Bush RM, (2003): Ecological and immunological determinants of influenza evolution. Nat..422.

Fitch WM, Bush RM. Bender CA, Cox NJ, (1977): Long term trends in the evolution of H(3) HAI human influenza type A. Proc. Nat. 1A. Cad Sci-USA. 94, 77-80. 
Food and Agricultural Organization, (2006): Vaccination of chickens against H5N1 avian influenza in the face of an outbreak interrupts virus transmission. Avi. Path.33, 405-412. Update in the avian influenza situation (As of 23/02/2006). FAO AIDE News.

Fouchier, R.A., Berkhoff, E.G., de Wit, E., Geelhoed-Mieras, M.M., Boon, A.C., Symons.J. Osterhaus, A.D., Rimmelzwaan, G.F., (2005): Functional constraints of influenza A virus epitopes limit escape from cytotoxic T lymphocytes. J. Virol.19. 11239-11246.

Gambaryan. A. S .,Tuzikov. A. B., Pazynina, G. V ., Webster, R. G., Matrosovich, M. N., and Bovio, N. Y. (2004): H5N1 chicken influenza viruses display a high binding affinity for Neu5Ac alpha 2-3 Gal beta 1-4(6-HS03) GlcNAc-containing receptors. Virol. 326(2), 310-316.

Gambaryan, A., Yamnikova, S ., Lvov, D., Tuzikov, A., Chinarcv, A ., Pazynina, G., Webster. R .,Matrosovich, M ., and Bovin, N. (2005): Receptor specificity of influenza viruses from birds and mammals: New data on involvement of the inner fragments of the carbohydrate chain. Virol. 334 (2). 276-283.

Garman. E., and Laver. G. (2004): Controlling influenza by inhibiting the virus's neuraminidase. Curr. Dr.Targ. 5(2). 119-136.

Garten RJ, Davis CT. Russell CA, Shu B. Lindstrom S. Balish A. Sessions WM, Xu X, Skepner E, Deyde V. (2009): Antigenic and genetic characteristics of swine-origin 2009 A (H1N 1) influenza viruses circulating in humans. Sci. 325, 197-201.

Gatherer, D, (2009): The 2009 HIN1 influenza outbreak in its historical context. J.Clin.Virol.45, 174-178.

Greninger. A.L., Chen, E.C.. Sittler, T., Scheinerman, A., Roubinian. N., Yu, G., Kim, E., Pillai, D.R., Guyard, C., Mazzulli, T., Isa, P., Arias, C.F.,Hackett. J ., Schochetman. G .. Miller, S., Tang, P., Chiu, C.Y., (20 I 0): A rneta genomic analysis of pandemic influenza A (2009 H1N1) infection inpatients from North America. PLoS ONE; 5, e 13381.

Guan, Y., Poon, L.L., Cheung, C.Y., Ellis. T.M., Lim, W., Lipatov, A.S., Chan, K.H., Sturm Ramirez, K.M., Cheung, C.L., Leung, Y.H., Yuen, K.Y (2000): Continuing evolution of H9 influenza viruses in Korean Poultry. 
Guilligay, D., Tarendeau, F.,Resa-Infante, P., Coloma, R., Crepin, T.. Sehr, P., Lewis, J., Ruigrok, R.W., Ortin, J., Hart, DJ., Cusack, S. (2008): The structural basis for cap binding by influenza virus polymerase subunit PB2. Nat.Struci.MolBiol.TS, 500-506.

Hale, B.G., Randall, R.E., Ortin, J ., Jackson, D., (2008): The multifunctional NS1 protein of influenza A viruses. J Gen. Virol.89. 2359-2376.

Hara, K., Schmidt, F.I., Crow, M., Brownlee, G.G., (2006): Amino acid residues in the Nterminal region of the PA subunit of influenza A virus RNA polymerase play a critical role in protein stability, endonuclease activity, cap binding and virion RNA promoter binding. J Virol. 80, 7789-7798.

Hatta. M., Gao, P., Halfmann, P., Kawaoka, Y .,(2001): Molecular basis for high virulence of Hong Kong H5N 1 influenza A viruses. Sci.293, 1840- 1842.

Hayden, F., Klirnov, A., Tashiro, M., Hay, A., Monto, A.. McKimm- Breschkin, J., Macken, C., Hampson, A., Webster, R.G., Amyard, M .. Zambon, M., (2005): Neurarninidase inhibitor susceptibility network position statement: antiviral resistance in influenza A/H5N1 viruses. Antivir. Ther.10, 873-877.

Holder. M and Lewis, P.O. (2003): Phylogeny estimation: traditional and Bayesian approaches. Nat. Rev. Genet. 4. 275-284.

Holmes EC. Ghedin E, Miller N, Taylor J, Bao Y. St George K. Grenfell BT. Salzberg SL Fraser CM. Lipman DJ, (2005): Whole-genome analysis of human influenza A virus reveals multiple persistent lineages and reassortment among recent H3N2 viruses. PLoS. Biol. 3, 300.

Honda, A., Mizumoto, K., Ishihama, A., (2002): Minimum molecular architectures for transcription and replication of the influenza virus. Proc. Nat. 1A. Cad. Sci. U S A. 99. 13 16613171.

Horimoto T, Kawaoka Y, (2001): Pandemic threat posed by avian influenza A viruses. Clin.Microbiol.Rev.14, 129-149.

Johnson NP, Mueller J, (2002): Updating the accounts: global mortality of the 1918-1920 "Spanish" influenza pandemic. Bull. Hist. med. 76. I 05-115. 
Karlsson Hedestam, G.B., Fouchier. R.A., Phogat, S .. Burton. D.R .. Sodroski. J., and Wyatt. R.T: (2008); A schematic presentation of influenza A virus.

Kilbourne, E., Johansson, B and Grajower, B. (1990): Independent and disparate evolution in nature of influenza A virus hemagglutinin and neuraminidase glycoproteins. Proceedings of the National Academy of Sciences of the United State of America. 87. 786-790.

Kim, J. A., Ryu, S. Y and Seo, S. H., (2005): Cells in the respiratory and intestinal tracts of chickens have different proportions of both human and avian influenza virus receptors. J Microbiol.43 (4), 366-369.

Koopmans, M., Wilbrink, B., Conyn, M., Natrop, G ., van der Nat, H., Vennema. H .. Meijer. A .,van Steenbergen, J., Fouchier, R., Osterhaus, A. and Bosman. A.(2004): Transmission of H7N7 avian influenza A virus to human beings during a large outbreak in commercial poultry farms in the Netherlands. Lancet.363 (9409), 587-593.

Krossoy B. Hordvik I, Nilsen F, Nylund A. Endresen C. The putative polymerase sequence of infectious salmon anemia virus suggests a new genus within the Orthomyxoviridae. .J Viral. 1999; 73: 2136-42.

Kuiken, T., Rimrnelzwaan, G., Van Riel, D., Van Amerongen, G., Baars, M ., Fouchier, R., and Osterhaus, A., (2004): Avian H5NI influenza in cats. Sci.306(5694), 241-241.

Lamb, R.A., Krug, R.M., (2001): Orthomyxoviridae: the viruses and their replication. In: Knipe, D.M., Howley, P. M. (Ed.) Fields Virol. Lippincott Williams and Wilkins, Philadelphia, Pp. 1487-1531.

Landolt GA, Olsen CW . (2007): Up to new tricks - a review of cross-species transmission of influenza A viruses. Animal health research reviews /Conference of Research Workers in Animal Diseases. 8: 1-21.

Li, M.L., Rao, P., Krug, R.M. (2001): The active sites of the influenza cap dependent endonuclease are on different polymerase subunits. EMBO.J20,2078-2086.

Luksza.M; Lassig, M. (2014): "A predictive fitness model for influenza". Nat. 507 (7490), 57- 61. doi:I0.1038/naturel3087. 
Matrosovich MN, Krauss S, Webster RG. (2001): H9N2 influenza A viruses from poultry in Asia have human virus-like receptor specificity. Virol.281:156-162.

Matrosovich, M.N.,Gambaryan, A. S., Tuzikov, A. B., Pazynina, G. V., Webster, R. G. and Bovin, N. V. (2004a): H5N1 chicken influenza viruses display a high binding affinity for Neu5Ac alpha 2-3Gal beta 1-4(6-HS03) GlcNAc-containing receptors. Virol.326(2), 310-3 16.

Matrosovich, M.N., Matrosovich, T.Y., Gray, T., Roberts, N.A., Klenk, H.D. (2004b): Neuraminidase is important for the initiation of influenza virus infection in human airway epithelium. J. Viro/.18 12665-12667.

McCown, M. F., and Pekosz A., (2006): Distinct Domains of the Influenza A Virus M2 Protein Cytoplasmic Tail Mediate Binding to the M1 Protein and Facilitate Infectious Virus Production. J Virol.80, 8178-8189.

Morens DM, Taubenberger JK, Fauci AS. (2009): The Persistent Legacy of the 1918 Influenza Virus. N. Engl. J.Med. 361, 225-229.

Munster VJ, Baas C, Lexmond P. Waldenstrom J. Wallensten A. Franssen T, Rimrnelzwaan GF, Beyer WE, Schutten M. Olsen B., (2007): Spatial, temporal, and species variation in prevalence of influenza A viruses in wild migratory birds. PLoSPathog. 3:e6 1

Munster VJ, Fouchier RA.,(2009): Avian influenza virus: of virus and birdecology. Vacc. 27:634 6344.

Neumann, G., Hughes, M.T., Kawaoka, Y., (2000): Influenza A virus NS2 protein mediates vRNP nuclear export through NES-independent interaction with hCRM1. EMBO. J. 19. 6751-6758.

Nobusawa.E. and Sato.K .(2006): Comparison of the mutation rates of human influenza A. and B.

Obayashi, E. Yoshida, H Kawai, F., Shibayama, N., Kawaguchi, A., Nagata, K., Tame, J.R., Park, S.Y.(2008): The structural basis for an essential subunit interaction in influenza virus RNA m polymerase. Nat.454, 1127- 1131.

Olsen CW., (2002): The emergence of novel swine influenza viruses in North America. Vir. 
Res.85: 199-210.

Olsen, B., Munster VJ., Wallensten, A. Waldenstrom, J .Osterhaus, A.O . Fouchier, R.A .. (2006): Global patterns of influenza a virus in wild birds. Sci.312, 384-388.

Ong AK, Hayden FG. John F., (2007): Enders lecture antivirals for influenza. J. Infect Dis. 196: 181-190.

Oxford JS., (2005): Preparing for the first influenza pandemic of the 21st century. Lancet. Infect. Dis.S, 129-3 1.

Palese P, Shaw ML. (2007): Orthomyxoviridae: the viruses and their replication. In: Knipe. D.M., Howley PM, editors. Fields Virol. Williams\& Wilkins: Philadelphia. Lippincott. Pp. 1647 $-1690$.

Pereda, A. (2010): Pandemic (HINI) 2009 outbreak on pig farm, Argentina.Emerg. Infect. Dis. 16. 304-307.

Perkins, L. E. L., and Swayne, D. E.. (2003): Comparative susceptibility of selected avian and mammalian species to a Hong Kong-origin H5N1 high-pathogenicity avian influenza virus. Avian Diseases. 47(suppln 3), 956-967.

Plotkin JB, Dushoff J, Levin SA. (2002): Hemagglutinin sequence clusters and the antigenic evolution of influenza A virus. Proc. Nat. La. Cad Sci USA. 23:23

Poole, E., Elton, D., Medcalf, L., Digard. P .(2004): Functional domains of the influenza A virus PB2 protein: identification of NP- and PB1-binding sites. Virol.321, 120-133.

Ravina, Manjeet, Mohan, H. et al. A changing trend in diagnostic methods of Influenza A (H3N2) virus in human: a review. 3 Biotech 11, 87 (2021). https://doi.org/10.1007/s13205-021-02642-w

Reperant LA, Rimmelzwaan GF, Kuiken T.(2009): Avian influenza viruses in mammals. Rev Sci Tech 28, 137-159.

Robert G. Webster, William J. Bean, Owen T. Gorman, Thomas M. Chambers,T and Yoshihiro Kawaoka (1992). Evolution and ecology of influenza a viruses. microbial. rev., Pp. 152-179 vol. 56, no. I 0146-0749/92/0I0152-28\$02.00/0. 
Ruigrok, R. W., Crepin. T., Hart D.L Cusack, S. (2010): Towards an atomic resolution understanding of the influenza virus replication machinery. Curr. Opin. Struct. Bio/ .20, 104-113.

Russell,R.J., Haire,L.F., Stevens.DJ., Collins.P.J., Lin,Y.P., Blackburn,G.M., Hay,A.J., Gamblin,S.J., and Skehel,J.J., (2006): The structure of H5N1 avian influenza neuraminidase suggests new opportunities for drug design. Nat. 443, 45-49.

Schweiger B. Schweiger, I. Zadow, and R. Heckler., (2002): Antigenic drift and variability of influenza viruses. Med. Microbial. Immunol.191(3-4): 133-138, Dec.

Skehel, J.J., Wiley, D.C. (2000): Receptor binding and membrane fusion in virus entry: the influenza hemagglutinin. Annu. Rev. Biochem.69, 531-569.

Smith, G.J.,Li, K.S., Guan, Y., Wang, J., Xu, K.M .. Duan, L., Rahardjo, A.P., Puthavathana, P., Buranathai, C., Nguyen. T.D., Estoepangestie, A.T.. Chaisingh, A., Auewarakul, P., Long, H.T., Hanh, N.T.. Webby, R.J., Poon, L.L., Chen. I-L Shortridge, K.F., Yuen, K.Y.. Webster, R.G., Peiris, J.S. (2004): Genesis of a highly pathogenic and potentially pandemic H5Nl influenza virus in eastern Asia. Nat.430, 209-213.

Smith, G.J., Vijaykrishna, D., Bahl, L Lycett, S.J., Worobey, M., Pybus, O.G.. Ma S.K., Cheung, C.L., Raghwani, J., Bhatt, S., Peiris, J.S., Guan, Y., Rarnbaut. A., (2009): Origins and evolutionary genomics of the 2009 swine origin H1N1 influenza A epidemic. Nat.459, 11221125.

Steinhauser, D. A., and J. J. Holland. (1987): Rapid evolution of RNA viruses. Am111. Rev. Microbial. 41,409-433.

Suzuki. T., Takahashi, T., Guo. C.T . Hidari, K.I., Miyamoto. D., Goto, H., Kawaoka. Y., Suzuki, Y., (2005): Sialidase activity of influenza A virus in an endocytic pathway enhances viral replication. J Virol.79, 11705-11715.

Suzuki, Yoshiyuki Suzuki., (2006): Natural selection on the influenza virus genome. Mol. Biol. Evol, 23(10): 1902- 1911.

Swayne DE. (2007): Understanding the complex pathobiology of high pathogenicity avian influenza viruses in birds. Avi. Dis. 51,242-249. 
Taubenberger JK, Morens DM., (2009): Pandemic influenza - including a risk assessment of H5N1. Rev Sci Tech. 28: 187-202 ..

Taubenberger JK, Reid AH, Lourens RM, Wang R, Jin G, Fanning TG. (2005): Characterization of the 1918 influenza virus polymerase genes. Nat. 437,889-893.

Taubenberger JK. (2006): Influenza hemagglutinin attachment to target cells: 'birds do it. we do it ... .. Fut. Virol. 1,415-418.

Taubenberger, J.K., Kash, J.C. (2010): Influenza virus evolution, host adaptation and pandemic formation. Cell Host Microbe 7, 440-451.

Taubenberger,J.K., Morens, D.M., Fauci. AS. (2009): The persistent legacy of the 1918 influenza virus. N Engl. J Med.361, 225-229.

Tong. S. (2012). A distinct lineage of influenza A virus from bats. Proc. Nat. La. cad. Sci. U S. A.109, 4269-4274.

Tong, S., Zhu. X .. Li, Y., Shi. M .. Zhang, J .. Bourgeois, M., Yang. H.. Chen. X., Recuenco. S., Gomez, J., Chen. L.-M., Johnson. A., Tao, Y., Dreyfus, C., Yu, W., McBride, R., Carney, P.J., Gilbert A.T., Chang, J., Guo, Z., Davis, C.T .. Paulson, J.C., Stevens, J., Rupprecht, C.E., Holmes. E.C., Wilson, T.A., Donis, R.O., (2013): New World Bats Harbor Diverse Influenza A Viruses. PLoS. PathogS, e I 003657.

Tumpey. T.M., Basler. C.F., Aguilar. P.V .. Zeng, H., Solorzano. A., Swayne. D.E., Cox, N.J., Katz, J.M .. Taubenberger, J.K., Palese, P., Garcia-Sastre. A. (2005): Characterization of the reconstructed 1918 Spanish influenza pandemic virus. Sci.310, 77-80. Van Hoeven, N., Pappas, C .,Belser, J.A., Maines, T.R., Zeng, H., Garcia- Sastre, A., Sasisekharan, R., Katz, J.M., Tumpey, T.M., (2009): Human HA and polymerase subunit PB2 proteins confer transmission of an avian influenza virus through the air. Proc. Nat. 1A. Cad Sci.US A. 106, 3366-3371.

Van Poucke SG, Nicholls JM, Nauwynck HJ, Van Reech K. (2010): Replication of avian, human and swine influenza viruses in porcine respiratory explants and association with sialic acid distribution. Virol.J. 7, 38. 
Vana, G; Westover, K. (2008): "Origin of the 1918 Spanish influenza virus: A comparative genomic analysis". Molecular Phylogenetics and Evolution. 47 (3): 1100 1110. doi: I 0.10 I 6/j.ympev.2008.02.003. PM ID I 8353690.

Viboud, C., Simonsen, L. (2012): Global mortality of 2009 pandemic influenza A H1N1.Lane.Infect Dis.12, 651-653.

Viboud, C; Boe/le, PY: Carrat. F; Val/eron. AJ; Flahault. A .. (2003): "Prediction of the spread of influenza epidemics by the method of analogues". Amer. J Epideniio. 158 (10). 96-1006. doi: I 0.1093/aje/kwg239.

Voeten, J. T. M., Bestebroer, T. M., Nieuwkoop, N. J., Fouchier. R. A. M., Osterhaus, A. D. M. E., and Rimmelzwaan, G. F., (2000). Antigenic Drift in the Influenza A Virus (H3N2) Nucleoprotein and Escape from Recognition by Cytotoxic T Lymphocytes. J. Virol. 74, 6800-6807.

Wang R, Soll L, Dugan V, Runstadler J, Happ G, Slemons RD, Taubenberger JK., (2008): Examining the hemagglutinin subtype diversity among wild duck-origin influenza A viruses using ethanol-fixed cloacal swabs and a novel RT-PCR method. Virol. 375, 182189.

Wang. P.. Song. W., Mok, B.W., Zhao, P., Qin, K., Lai, A., Smith. G.J., Zhang, J .. Lin, T.. Guan, Y., Chen, H., (2009): Nuclear factor 90 negatively regulates influenza virus replication by interacting with viral nucleoprotein. J. Virol.83, 7850- 7861.

Webster RG, Hulse-Post DJ. Sturm-Ramirez K.Jtt. Guan Y. Peiris M. Smith G, Chen H., ('.2007): Changing epidemiology and ecology of highly pathogenic avian HSN I influenza viruses. Avi. Dis. 51. 269-272.

Webster, R. G., and Hulse, D. J., (2004): Microbial adaptation and change: avian influenza. Scientific and Technical Review, World Organisation for Animal Health.23(2), 453-465.

Webster, R. G., Peiris. M., Chen. J-T. L., and Guan, Y. (2006): HSN I outbreaks and enzootic influenza. Emerg. Inject Dis. 12(1), 3-8. 
World Health Organization(2005): Avian influenza A (H5NI) infection in humans. N. Engl. J Med. 353, 1374-1385.

World Health Organization. (2009): World now at the start of 2009 influenza pandemic (WHO\}. Wright PF, Neumann G, Kawaoka Y (2007): Orthomyxoviruses. In: Knipe DM, Howley PM editors. Fields Virol. Williams \& Wilkins: Philadelphia, Lippincott: 2007. Pp. 1691--1740.

Wright, P. F., and Webster, R. G. (2001):Orthomyxoviruses. In: Fields Virology. fourth edition, Knipe DM, Howley PM eds, Lippincott, Philadelphia. Pp. 1533-1579. 